\title{
Insular glioma surgery: an evolution of thought and practice
}

\author{
JNSPG 75th Anniversary Invited Review Article
}

\author{
Shawn L. Hervey-Jumper, MD, and Mitchel S. Berger, MD \\ Department of Neurological Surgery, University of California, San Francisco, California
}

OBJECTIVE The goal of this article is to review the history of surgery for low- and high-grade gliomas located within the insula with particular focus on microsurgical technique, anatomical considerations, survival, and postoperative morbidity.

METHODS The authors reviewed the literature for published reports focused on insular region anatomy, neurophysiology, surgical approaches, and outcomes for adults with World Health Organization grade II-IV gliomas.

RESULTS While originally considered to pose too great a risk, insular glioma surgery can be performed safely due to the collective efforts of many individuals. Similar to resection of gliomas located within other cortical regions, maximal resection of gliomas within the insula offers patients greater survival time and superior seizure control for both newly diagnosed and recurrent tumors in this region. The identification and the preservation of $\mathrm{M}_{2}$ perforating and lateral lenticulostriate arteries are critical steps to preventing internal capsule stroke and hemiparesis. The transcortical approach and intraoperative mapping are useful tools to maximize safety.

CONCLUSIONS The insula's proximity to middle cerebral and lenticulostriate arteries, primary motor areas, and perisylvian language areas makes accessing and resecting gliomas in this region challenging. Maximal safe resection of insular gliomas not only is possible but also is associated with excellent outcomes and should be considered for all patients with low- and high-grade gliomas in this area.

https://thejns.org/doi/abs/10.3171/2018.10.JNS181519

KEYWORDS anaplastic astrocytoma; glioblastoma; low-grade glioma; insula; insular glioma; oligodendroglioma; brain mapping; oncology

$\mathrm{T}$ HE insular cortex was first named by German neurologist J. C. Reil in 1809 and is a common location for glial tumors. ${ }^{34}$ Gliomas within the insula have historically been challenging to manage due to the complex shape and organization of the insular cortex, the insula's functional significance, and its intimate relationship with the internal cerebral artery, the middle cerebral artery, and lenticulostriate vessels. Given these important considerations, prior to the current era, insular gliomas were considered too dangerous for surgical treatment, with an unacceptably high rate of postoperative morbidity. However, over the past 2 decades, large-scale molecular characterization studies, combined with a more complete understanding of the role of cytoreductive surgery for newly diagnosed and recurrent gliomas, have illustrated the importance of maximal extent of resection (EOR) to enhance overall and progression-free survival for all patients. ${ }^{5,22,33,38}$ Neurosurgeons have therefore been compelled to develop treatment paradigms for low- and highgrade gliomas located within the insular cortex. Although there remain many unknowns about the function of the normal insula, it is known that resection of gliomas in this region can be accomplished with a low morbidity profile. Efforts to reduce perioperative morbidity have come about through careful study employing anatomical dissections, structural and functional imaging analysis, and language,

ABBREVIATIONS EOR = extent of resection; IFOF = inferior frontal occipital fasciculus; WHO = World Health Organization.

SUBMITTED September 28, 2018. ACCEPTED October 1, 2018.

INCLUDE WHEN CITING DOI: 10.3171/2018.10.JNS181519. 
motor, and cognition mapping, in addition to meticulous prospective and retrospective assessment of patient outcomes and long-term morbidity. ${ }^{10,12}$ In this article, we review the published literature to offer a historical perspective on surgery for patients with low- and high-grade gliomas within the insula.

\section{Anatomy and Function of the Insula Neuroanatomy}

Due to an extensive network of afferent connections, the insula has been implicated in a variety of sensory, motor, emotional, and cognitive functions. These associations early on made some authors argue that the insular cortex is functionally unique, providing the underpinning for behavioral and cognitive foundations exclusive to humans. ${ }^{1,16}$ The anatomical location of the triangle-shaped insula, protected within the folds of the sylvian fissure, may have added to the mystique and inspired the name "insula," which is Latin for island ("Island of Reil"; also known as the hidden fifth lobe of the brain). The insular cortex is covered by the frontal, temporal, and parietal lobes (Fig. 1A). The insular surface faces laterally and is enclosed by the anterior and posterior limiting (also known as circular) sulci. ${ }^{35}$ The limiting sulcus has anterior, superior, and inferior parts. Grossly, the insula is composed of an anterior limen insula and is divided by a central sulcus into anterior and posterior parts. Two anterior sulci separate the 3 short gyri, and a single sulcus separates the 2 long posterior gyri. The central sulcus of the insula is in line with the central sulcus of the cerebral hemispheres, separating the frontal and parietal lobes. The insular apex is the highest and most prominent laterally projecting area on the insular convexity. Lying underneath the cortical surface of the pars opercularis is the superior portion of the anterior and middle short insular gyri (Fig. 1A). Posteriorly, the supramarginal gyrus overlies the superior limiting sulcus and the superior portion of the posterior long gyri (Fig. 1A). The limen insula overlies the uncinate fasciculus. ${ }^{6,44}$ Additionally, the anterior perforated substance lies medial to the limen. Positioned deep to the insular cortex are the extreme capsule, claustrum, putamen, and external capsule, followed by the anterior and posterior genu of the internal capsule (Fig. 1B).

The insular cortex has a rich vascular supply, extending from the internal cerebral and middle cerebral arteries (Fig. 2). The middle cerebral artery bifurcates at the limen insula, forming between 1 and 6 insular $\mathrm{M}_{2}$ branches, which overlie the insular surface. Insular arteries supply the insular cortex, extreme capsule, claustrum, and external capsule. The lateral lenticulostriate arteries supply the internal capsule, putamen, and globus pallidus. ${ }^{45,46}$ Microscopically, there is progressive loss of granular layer 4, resulting in 3 cytoarchitectural insular areas: granular, dysgranular, and agranular sections. The granular layer has a classic 6-layered cortical structure. Layer 4 becomes thinner in the dysgranular insula and disappears entirely in the agranular insula. Cortical layer 5 is, however, marked by large bipolar neurons with unknown function, which have been documented historically by Ramón y Cajal and Constantin von Economo. ${ }^{16}$
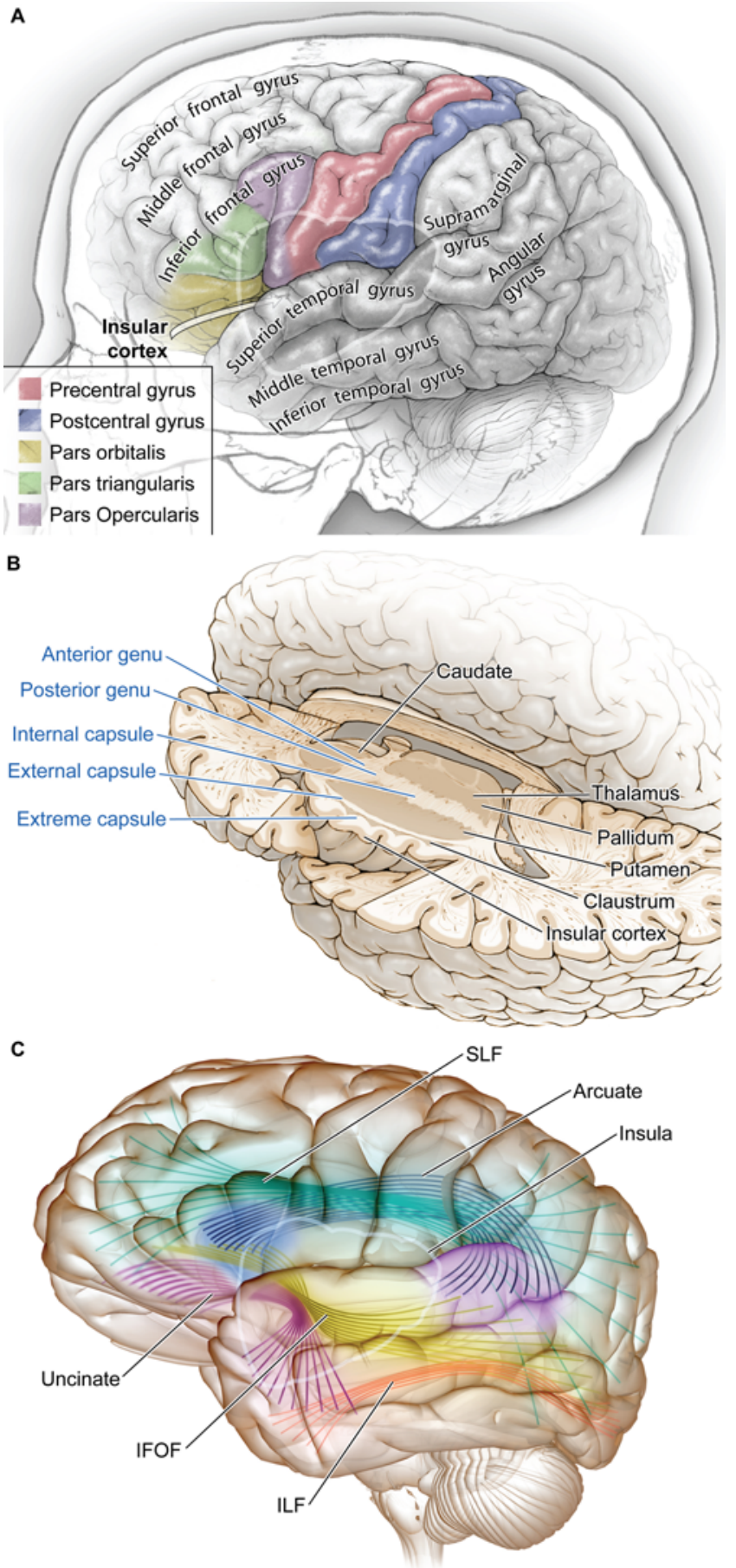

FIG. 1. Anatomical illustration of the lateral cortical surface. A: The insula is covered by the frontal, parietal, and temporal lobes. The pars triangularis (green), pars opercularis (purple), and precentral gyrus (red) overlie the anterior aspect of the insula. B: Cross-sectional image of cadaveric dissection illustrating the insula with underlying extreme capsule, claustrum, external capsule, putamen, globus pallidus, internal capsule, and thalamus. C: Lateral cortical projection illustrating dorsal and ventral diffusion tensor imaging language tracts. Both the IFOF and uncinate fasciculi project through the insula. ILF = inferior longitudinal fasciculus; SLF = superior longitudinal fasciculus. Artist: Kenneth Probst. Copyright Department of Neurological Surgery, University of California, San Francisco. Published with permission. 


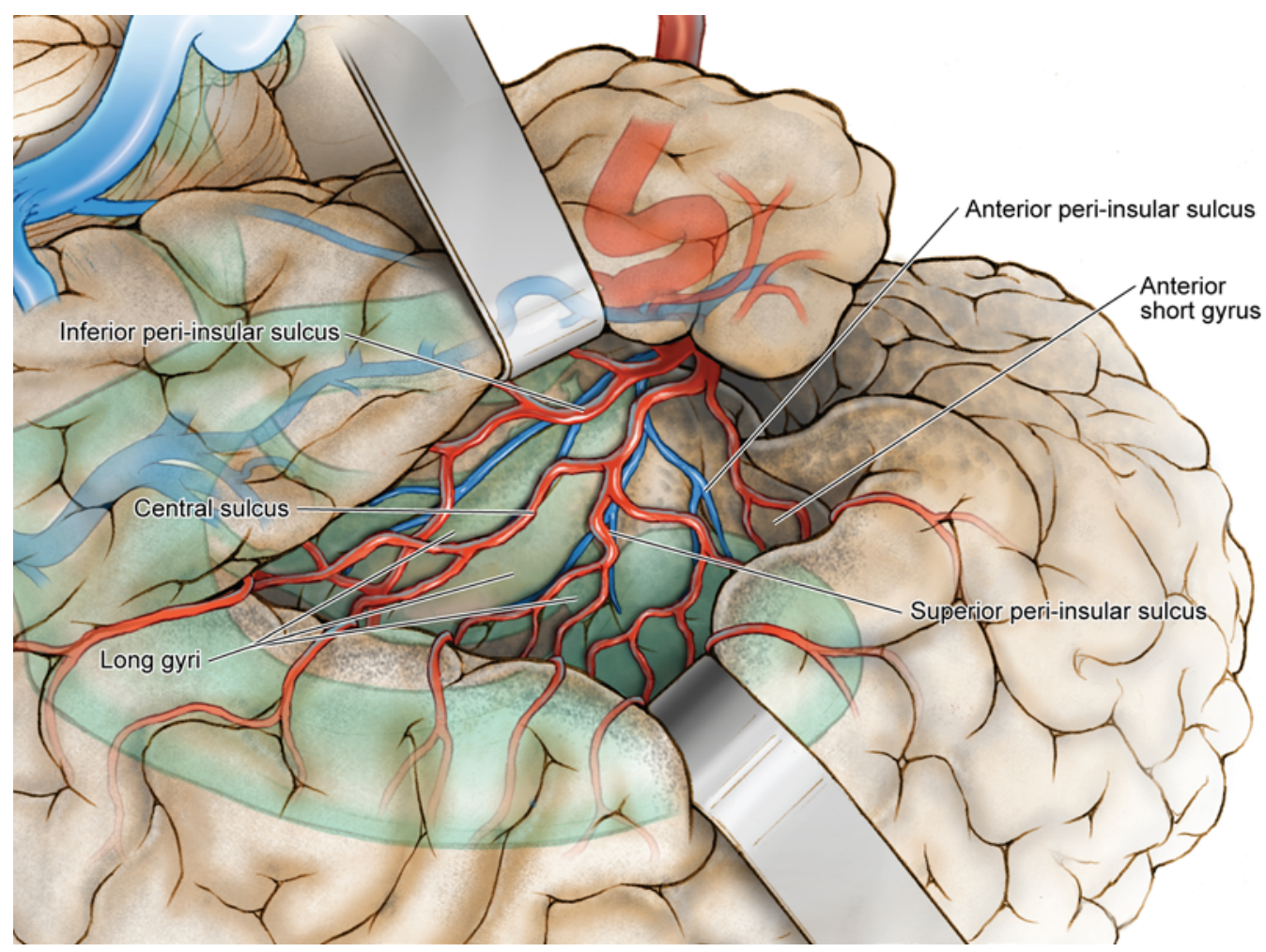

FIG. 2. Cadaveric dissection with surgeon's view. Both anterior short and posterior long gyri are visible with overlying long $M_{2}$ insular perforators. The central sulcus of the insula is in line with the central sulcus of the cerebral cortex. Artist: Kenneth Probst. Copyright Department of Neurological Surgery, University of California, San Francisco. Published with permission.

\section{Network Connectivity}

Large-scale network connections within the dominant and nondominant insula suggest wide-ranging functional significance, including sensory, limbic, and cognitive modalities. A large portion of the insula is dedicated to receiving sensory input from both thalamic and cortical afferents, which carry olfactory, gustatory, visual, auditory, and somatosensory information. ${ }^{16}$ Together, sensory projections are topographically organized along the insular cortex, but because no modality is purely sensory, the insular cortex can best be thought of as a multimodal integration site. In addition to sensory input, projections from the orbitofrontal and cingulate regions are implicated in reward, drive, and motivation. Connections with the limbic system, particularly the central amygdala, dorsomedial thalamus, hypothalamus, stria terminalis, and parahippocampal gyrus, suggest a role in fear and anxiety. 1,3,27

\section{Functional Significance}

Integrated sensory inputs from the thalamus and afferents from areas implicated in emotional processing converge in the insula and subcortical white matter. Functional implications of the insula for human behavior are based almost entirely on functional MRI activation during the administration of assorted tasks and pharmacological lesional studies in rats, both of which have failed to provide a direct causal link that ties specific loci within the insula to function and behavioral change. Topographically organized afferents from the thalamus carry information from many bodily sensations, such as blood oxygenation, carotid baroreceptors, blood pressure, hunger, pain, and thirst. It is therefore believed that the insula functions to integrate autonomic sensory input. ${ }^{27}$ This includes both positive and negative emotions, such as fear, anxiety, sadness, joy, anger, and disgust. In healthy subjects, experiments applying Pavlovian fear conditioning illustrate insula activation; however, it remains unclear whether the insula's role is primarily involved in fear promotion or fear inhibition. ${ }^{16}$ Given the multimodal sensory and limbic afferents, the insular cortex is thought to be partially responsible for assigning emotional relevance to sensory stimuli. One important but rare example of this anatomical consideration is illustrated in case reports of insula lesioning following stroke, in which patients lacked taste and flavor recognition. ${ }^{16}$ Similarly, pain dissociation has been documented to occur following stroke, in which patients recognize pain but lack an appropriate negative response to the stimulus. ${ }^{16}$ Interestingly, the anterior insula is active in functional MRI studies when a person observes emotion such as pain in another person, suggesting that the insular cortex contributes to the processing of empathy. Classically, the insula has been implicated in speech and language processing. Both the uncinate fasciculus and the inferior frontal occipital fasciculus (IFOF) extend through the insula subcortical region (Fig. 1C) ${ }^{20,28}$ The uncinate fasciculus extends from the inferior frontal gyrus, under the Sylvian fissure, terminating in the superior temporal gyrus, while the IFOF extends posteriorly toward the oc- 
cipital pole (Fig. 1C). For this reason, subcortical intraoperative brain mapping of language tasks is essential during dominant-hemisphere insular glioma surgery. ${ }^{24}$

\section{Surgery for Insular Tumors Prior to the Modern Era}

The argument in support of maximal EOR has evolved over the past 20 years. These efforts began with gross estimates of EOR and volume of residual tumor based on radiology reports separating patients into "subtotal" and "gross-total" resection cohorts. The next wave of studies subsequently employed cross-sectional measures of tumor volume based on the lesion's maximal diameter. This approach led to volumetric measures of EOR. ${ }^{41}$ Given the 2016 World Health Organization (WHO) subclassification of gliomas based on molecular features, present investigations weigh the impact of volumetric EOR measures across molecular subgroups, distinguishing, for example, between oligodendroglioma and astrocytoma and isocitrate dehydrogenase 1/2 mutant versus wild-type tumors. ${ }^{5,7,17,23,26,49}$ Throughout the evolution of this ongoing discussion, EOR for gliomas within the insula has remained a controversial subject. Early on, despite evidence in favor of maximal safe resection, few attempts were made to remove insular gliomas because practitioners believed that surgery in the insula posed too great a risk. Even so, several reports between 2000 and 2009 demonstrated the possibility of insular glioma surgery, chronicling technical considerations and complication rates. ${ }^{11-14,19,25,29,31,32,39,47,51-53}$

Yaşargil et al. first described the transsylvian approach via the pterional craniotomy for 240 patients with tumors of the limbic and paralimbic regions, including the insula. A histological summary of cases and tumor volumes was not provided, but the authors reported seizures as the most common presenting symptom. In their series, $95 \%$ of patients experienced "minimal" neurological deficits, with the ability to function independently and eventually return to work over an unknown period of time. These results demonstrated that a microsurgical approach to insular tumors could be considered. This led Yaşargil et al. to subsequently propose a classification system based on whether a tumor was restricted to the insula (type 3), was part of the insula (type 3A), or was included in the adjacent operculum (type 3B). ${ }^{21,51,52}$ In this classification system, insular lesions involving one or both of the paralimbic orbitofrontal and temporopolar areas were classified, respectively, as type 5A or type 5B. ${ }^{21,51,52}$ Though comprehensive, this classification system was ineffective at addressing some of the anatomical features relevant to surgery for insular gliomas, such as proximity to potentially functional areas. Ebeling and Kothbauer then reported their experience treating 5 patients (ages 26-51 years) with circumscribed low-grade astrocytomas involving the dominanthemisphere frontal operculum and insular region. Only one patient in this pilot series had a predominantly insular tumor, and the authors chose a transsylvian approach, with intraoperative motor mapping of the frontal operculum. Surgery for this patient was abruptly halted due to sudden contralateral hemiparesis, which was presumed to be secondary to an internal capsule stroke. The overall complication rate for this series was $20 \% .{ }^{14}$ Based on their postoperative neurological outcomes, the authors advocated for biopsy only in cases of large dominant-hemisphere insular and opercular-insular gliomas. ${ }^{14}$

Improvements in microsurgical technique, the more widespread use of cortical and subcortical intraoperative language and motor mapping, and superior neuroanesthesia have contributed to subsequent series reporting improved patient outcomes. Zentner et al. and Vanaclocha et al. reported their experience treating, respectively, 30 and 23 patients with insular gliomas in whom there was a roughly equal distribution of gliomas restricted to the insula and tumors extending into the frontal or temporal operculum. In these series, the authors were able to achieve gross-total resection in $16 \% \%^{53}$ and $86 \%{ }^{47}$ of cases. Sixty-three percent of patients experienced immediate postoperative neurological deficits, including hemiparesis and stroke, which improved to a $10 \%-22 \%$ morbidity rate after a mean follow-up period of 8.5 months. ${ }^{47,53}$ In the next wave of studies, surgeons meticulously dissected anatomical structures and described surgical techniques to maximize safety. ${ }^{11,25}$ Recommendations included the following: 1) wide splitting of the sylvian fissure, 2) awake craniotomy with cortical and subcortical mapping to identify the overlying motor cortex tract and the internal capsule subcortically, and 3) meticulous suprasylvian dissection to avoid coagulation of the long perforating $\mathrm{M}_{2}$ segment and lateral lenticulostriate

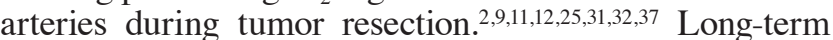
3-month neurological complication rates subsequently dropped to $8 \%-10 \% .11,19,25$

\section{Surgery for Insular Tumors in the Modern Era}

While detailed reports outlining microsurgical techniques addressed how insular gliomas could be approached, many important questions remained. Does treatment with maximal resection in patients with insular gliomas confer a survival benefit similar to that of patients with tumors in less risky regions? Does the location of the tumor within the insula impact perioperative morbidity? Are neurosurgeons able to predict EOR and perioperative morbidity based on the anatomical position of the lesion? Are there quality of life, seizure control, and functional outcome limitations experienced by patients? Simon et al. were the first to report on a large series of patients with WHO grade II-IV insular glioma. ${ }^{39}$ In their series, EOR above $90 \%$ was achieved in $42 \%$ of cases, and EOR of 70\%-90\% was accomplished in $51 \%$ of cases. Predictors of poor outcome included WHO grade IV glioblastomas, advanced age, and low preoperative Karnofsky Performance Scale score. Predictors of "favorable" outcome after univariate analysis included younger age at diagnosis (< 40 years); WHO grade I, II, and III histology; Yaşargil type 5A/B tumors that extend into the frontal operculum; and an EOR $>90 \% .{ }^{39}$ Interestingly, the median survival for patients with WHO grade III anaplastic astrocytomas was 5 years, and the 5-year survival rate for patients with anaplastic oligodendrogliomas was $80 \% .^{39}$ Sanai et al. published a longitudinal study of 115 surgical procedures involving 104 patients with insular gliomas in order to better understand the relationship between EOR and survival, malignant transformation, and perioperative 


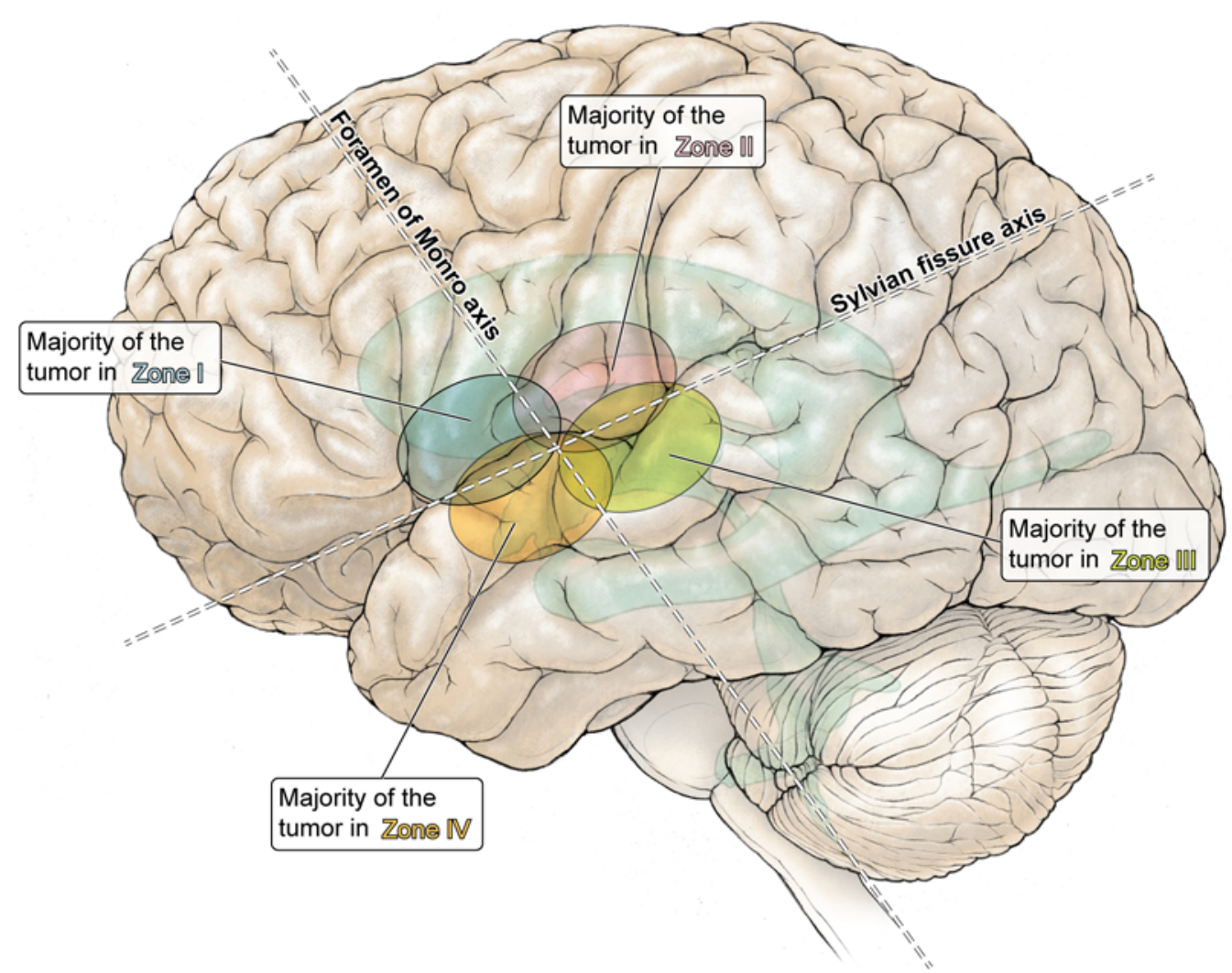

FIG. 3. The Berger-Sanai classification of insular gliomas separates insular gliomas based on their location above or below the sylvian fissure and anterior or posterior to the foramen of Monro. Zone 1 gliomas are located above the sylvian fissure, anterior to the foramen on Monro. Zone 2 gliomas are superior to the sylvian fissure and posterior to the foramen of Monro. Zone 3 gliomas are positioned inferior to the sylvian fissure and posterior to the foramen of Monro. Zone 4 gliomas are inferior to the sylvian fissure and anterior to the foramen of Monro. Artist: Kenneth Probst. Copyright Department of Neurological Surgery, University of California, San Francisco. Published with permission.

morbidity.$^{38}$ At that time, the literature lacked a clinically useful nomenclature covering the relevant anatomical considerations learned over the prior decade. The Berger-Sanai classification system was therefore introduced, dividing the insular cortex into quadrants based on the sylvian fissure and foramen of Monro. ${ }^{6,21,38}$ The insula was divided into 4 zones (Fig. 3). Along the horizontal plane in a sagittal view, the insula straddles the sylvian fissure. This plane is intersected by a perpendicular line at the foramen of Monro. Tumor location was assigned to one or more of these zones. Zone 1 (anterosuperior) was the most common site, with $35 \%$ of insular gliomas occurring within this zone. Zone 2 (posterosuperior) tumors represented 6\% of cases, extending above the sylvian fissure and behind the foramen of Monro. The precentral gyrus and primary motor cortex are important anatomical considerations for this zone. Zone 3 (inferoposterior) tumors represented another $6 \%$ of cases, and these tumors may extend into Heschl's gyrus. Another $6 \%$ of insular gliomas were predominantly within zone 4 (inferoanterior) below the sylvian fissure and anterior to the foramen of Monro. It is important to consider subcortical mapping for identification of the internal capsule and corticospinal tract during resection of zone 4 tumors. Using this classification scheme, nearly $40 \%$ of tumors were equally represented within 2 zones, and $13 \%$ were consid- ered giant, extending into all 4 quadrants. ${ }^{38}$ The BergerSanai classification scheme has remained the most widely used tool, with high interuser reliability, as demonstrated by a Kappa coefficient of $0.86 .{ }^{21}$

Regardless of zone, recent reports suggest a median EOR of $81 \%-86 \%$ for low- and high-grade insular gliomas. ${ }^{15,21,38,40}$ Zone 1 gliomas have the highest median EOR rate $(86 \%){ }^{38}$ Patients with WHO grade II insular gliomas with $>90 \%$ EOR have a 5-year overall survival rate of $100 \%$, while those with $<90 \%$ EOR have a 5-year overall survival rate of $84 \%$. Similarly, patients with WHO grade III and IV insular gliomas with an EOR > 90\% have a 2 -year overall survival rate of $91 \%$, but when the EOR is $<90 \%$, the overall survival rate drops to $75 \% .^{38}$ Most importantly, EOR for insular gliomas is predictive of both overall and progression-free survival for WHO grade II, III, and IV tumors. ${ }^{21,38}$ Additionally, patients with a lower EOR had greater rates of malignant transformation. ${ }^{38}$ Therefore, reoperation at the point of tumor recurrence is a consideration that has recently been addressed. The EOR during reoperation is not impacted by the BergerSanai zone. Following reoperation for recurrent insular glioma, $91 \%$ of patients have no new postoperative deficits at 3 months, which is similar to results for newly diagnosed patients. ${ }^{30}$ 

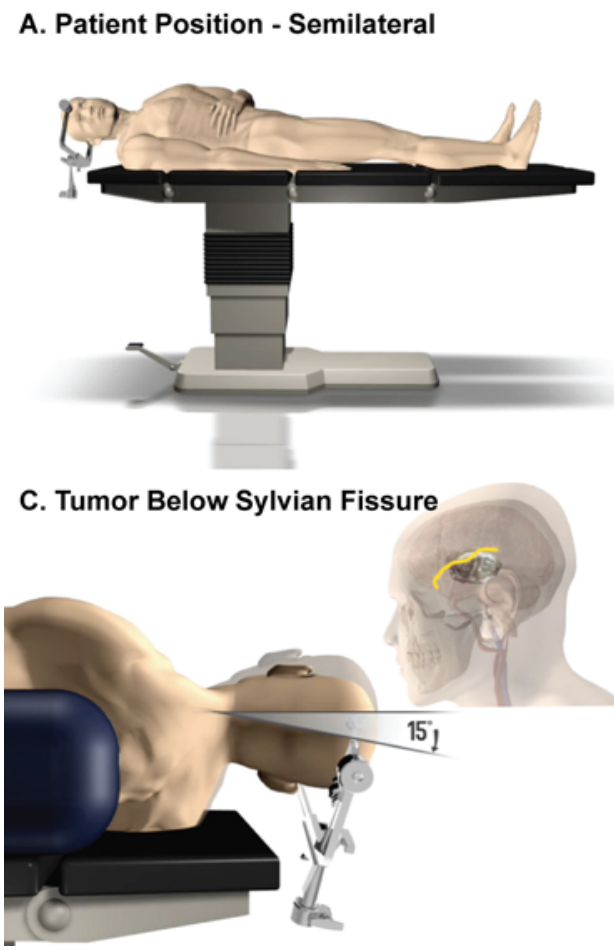

\section{B. Zone $2 \& 3$ Tumors}

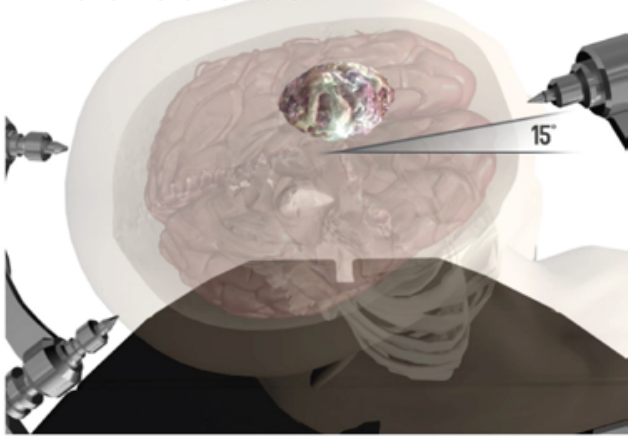

D. Tumor Above Sylvian Fissure

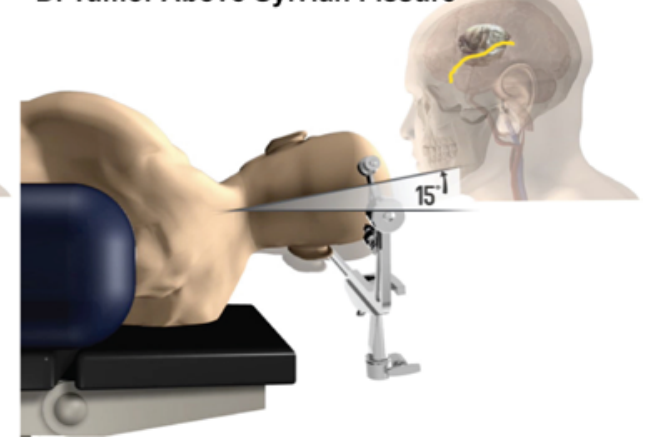

FIG. 4. A: Patients are positioned semilateral. B: When approaching insular gliomas located behind the foramen of Monro (zones 2 and 3 ), the head is positioned $15^{\circ}$ upward to maximize visualization underneath functional cortical areas. For tumors positioned below the sylvian fissure, the vertex of the head is tipped $15^{\circ}$ toward the floor $(\mathbf{C})$, while for tumors located above the sylvian fissure, the vertex of the head is tipped $15^{\circ}$ toward the ceiling (D). Artist: Kenneth Probst. Copyright Department of Neurological Surgery, University of California, San Francisco. Published with permission.

Perioperative morbidity has remained low and no deaths related to surgery have been reported in the recent literature. Awake craniotomy with cortical and subcortical stimulation mapping results in a lower rate of permanent neurological deficits compared with insular gliomas approached without mapping (19\% neurological impairment rate for patients without mapping vs $4.3 \%$ with mapping, $\mathrm{p}=0.008){ }^{2,18}$ Furthermore, patients with WHO grade III-IV gliomas are more likely to experience long-term neurological deficits than those with WHO grade II gliomas. ${ }^{42,45,49,51}$ With respect to location, short-term neurological complications occur most frequently following resection of zone 1 tumors and giant insular gliomas involving all 4 zones. While the immediate (within 3 days of surgery) short-term complication rate is $20 \%-26 \%$, after 3 months, the overall complication rate is 3.2\%-9\% across published reports. ${ }^{21,36,38}$ New motor neurological deficits occur in $7.8 \%-9 \%$ of patients, resolving to $1.6 \%$ within 3 months. ${ }^{21,38}$ Postoperative language deficits occur in $16.3 \%$ of patients immediately following surgery, resolving to $0.8 \%$ within 3 months. ${ }^{21,38}$ In addition to brain mapping, studies focused on the use of intraoperative assistive technologies such as navigated 3D ultrasound and high-field intraoperative MRI have demonstrated utility, particularly with respect to minimizing postoperative morbidity., $, 8,43,54$

The surgical approach to insular gliomas has been revisited in the past decade. Patients are placed in a semilateral position with head parallel to the floor. For the patient with a lesion located in zone 2 or 3 behind the foramen of Monro, the head can be rotated $15^{\circ}$ upward, allowing tumor resection beneath functional cortical structures positioned over the posterior insula (Fig. 4). The vertex of the head is then tipped $15^{\circ}$ toward the floor or $15^{\circ}$ toward the ceiling, based on whether the tumor is located above or below the sylvian fissure ${ }^{38}$ Dating back to Yaşargil's early publications, the most commonly described route to the insula was the transsylvian approach, splitting the fissure. However, a dominant draining vein is found crossing the sylvian fissure, requiring sacrifice for larger exposures in up to $87 \%$ of cases. ${ }^{6}$ An alternative transcortical approach has been described in which the overlying frontal, temporal, and parietal cortices are mapped for sites of language, motor, and cognitive significance. Cortical windows are then created through the operculum, exposing the insular cortex and long $\mathrm{M}_{2}$ perforators (the "cortical window approach"). ${ }^{6,21}$ Working through these windows, the insular component of the tumor is resected. Critical steps include identification of the lenticulostriate arteries, which mark the medical border of the resection. Subcortical mapping permits the identification of the corticospinal tract within the internal capsule. The transopercular cortical window approach is particularly useful for large tumors in all 4 zones because it provides maximal insular exposure and preservation of the bridging veins. ${ }^{6,36}$

Functional outcomes, morbidity, and quality of life remain important considerations when assessing patients with insular gliomas. The insular cortex receives extensive somatosensory, limbic, and cognitive afferent input, yet 
patients are able to experience a high quality of life and minimal morbidity following surgery in this region. ${ }^{50}$ Seizure control is one important factor for quality of life considerations, particularly given that the majority of patients present with seizures. In addition to offering a survival benefit, maximizing EOR for insular gliomas improves seizure control. ${ }^{48}$ Furthermore, recurrence of seizures often predicts glioma recurrence and progression. ${ }^{48}$ Quality of life, as it relates to language outcomes, is also an important consideration. Despite the uncinate fasciculus and IFOF extending through the insula and the superior longitudinal fasciculus and arcuate fasciculus extending in superior and posterior directions, awake language mapping allows for the surgeon to identify language sites in this critical region (Fig. 1). Language tasks applied during insular glioma resection include picture naming, text reading, 4-syllable repetition, auditory naming, and syntax production. The majority of patients with dominant-hemisphere low-grade insular gliomas have excellent baseline language task performance, suggesting that language plasticity might play a role. ${ }^{12}$ Disability due to severe emotional, mood, autonomic, and personality concerns is rarely observed in patients with insular gliomas either at presentation or following resection. This raises the question of whether thalamic and limbic afferent pathways to the insula are functionally relevant or whether central nervous system plasticity and functional reorganization contribute to these observations. ${ }^{13,42}$

\section{Conclusions}

The insula's proximity to the middle cerebral and lenticulostriate arteries, primary motor areas, and the perisylvian language network makes accessing and resecting gliomas in this area challenging. The collective contributions of many surgeons, anesthesiologists, anatomists, and behavioral neuroscientists over more than 20 years have shed light on the anatomy and function of this important brain region. Enhanced microsurgical techniques and awake language and motor mapping have been the driving force behind improved patient outcomes. Maximal safe resection of insular gliomas continues to be associated with improved patient outcomes and should be considered for all patients with low- and high-grade gliomas.

\section{Acknowledgments}

We would like to thank Melissa Lau, $\mathrm{PhD}$, for her assistance with technical editing for this manuscript.

\section{References}

1. Accolla R, Carleton A: Internal body state influences topographical plasticity of sensory representations in the rat gustatory cortex. Proc Natl Acad Sci U S A 105:4010-4015, 2008

2. Alimohamadi M, Shirani M, Shariat Moharari R, PourRashidi A, Ketabchi M, Khajavi M, et al: Application of awake craniotomy and intraoperative brain mapping for surgical resection of insular gliomas of the dominant hemisphere. World Neurosurg 92:151-158, 2016

3. Augustine JR: Circuitry and functional aspects of the insular lobe in primates including humans. Brain Res Brain Res Rev 22:229-244, 1996
4. Barbosa BJ, Dimostheni A, Teixeira MJ, Tatagiba M, Lepski $\mathrm{G}$ : Insular gliomas and the role of intraoperative assistive technologies: results from a volumetry-based retrospective cohort. Clin Neurol Neurosurg 149:104-110, 2016

5. Beiko J, Suki D, Hess KR, Fox BD, Cheung V, Cabral M, et al: IDH1 mutant malignant astrocytomas are more amenable to surgical resection and have a survival benefit associated with maximal surgical resection. Neuro Oncol 16:81-91, 2014

6. Benet A, Hervey-Jumper SL, Sánchez JJ, Lawton MT, Berger MS: Surgical assessment of the insula. Part 1: surgical anatomy and morphometric analysis of the transsylvian and transcortical approaches to the insula. J Neurosurg 124:469-481, 2016

7. Cahill DP, Beiko J, Suki D, Prabhu SS, Weinberg J, Lang FF, et al: IDH1 status and survival benefit from surgical resection of enhancing and nonenhancing tumor in malignant astrocytomas. J Clin Oncol 30 (15 Suppl):2019, 2012 (Abstract)

8. Chen LF, Yang Y, Ma XD, Yu XG, Gui QP, Xu BN, et al: Optimizing the extent of resection and minimizing the morbidity in insular high-grade glioma surgery by high-field intraoperative MRI guidance. Turk Neurosurg 27:696-706, 2017

9. Duffau H: A personal consecutive series of surgically treated 51 cases of insular WHO Grade II glioma: advances and limitations. J Neurosurg 110:696-708, 2009

10. Duffau H: Surgery of low-grade gliomas: towards a 'functional neurooncology'. Curr Opin Oncol 21:543-549, 2009

11. Duffau H, Capelle L, Lopes M, Faillot T, Sichez JP, Fohanno D: The insular lobe: physiopathological and surgical considerations. Neurosurgery 47:801-811, 2000

12. Duffau H, Moritz-Gasser S, Gatignol P: Functional outcome after language mapping for insular World Health Organization Grade II gliomas in the dominant hemisphere: experience with 24 patients. Neurosurg Focus 27(2):E7, 2009

13. Duffau H, Taillandier L, Gatignol P, Capelle L: The insular lobe and brain plasticity: lessons from tumor surgery. Clin Neurol Neurosurg 108:543-548, 2006

14. Ebeling U, Kothbauer K: Circumscribed low grade astrocytomas in the dominant opercular and insular region: a pilot study. Acta Neurochir (Wien) 132:66-74, 1995

15. Eseonu CI, ReFaey K, Garcia O, Raghuraman G, QuinonesHinojosa A: Volumetric analysis of extent of resection, survival, and surgical outcomes for insular gliomas. World Neurosurg 103:265-274, 2017

16. Gogolla N: The insular cortex. Curr Biol 27:R580-R586, 2017

17. Gozé C, Rigau V, Gibert L, Maudelonde T, Duffau H: Lack of complete 1p19q deletion in a consecutive series of 12 WHO grade II gliomas involving the insula: a marker of worse prognosis? J Neurooncol 91:1-5, 2009

18. Gravesteijn BY, Keizer ME, Vincent AJPE, Schouten JW, Stolker RJ, Klimek M: Awake craniotomy versus craniotomy under general anesthesia for the surgical treatment of insular glioma: choices and outcomes. Neurol Res 40:87-96, 2018

19. Hentschel SJ, Lang FF: Surgical resection of intrinsic insular tumors. Neurosurgery 57 (1 Suppl):176-183, 2005

20. Herbet G, Moritz-Gasser S, Duffau H: Direct evidence for the contributive role of the right inferior fronto-occipital fasciculus in non-verbal semantic cognition. Brain Struct Funct 222:1597-1610, 2017

21. Hervey-Jumper SL, Li J, Osorio JA, Lau D, Molinaro AM, Benet A, et al: Surgical assessment of the insula. Part 2: validation of the Berger-Sanai zone classification system for predicting extent of glioma resection. J Neurosurg 124:482488, 2016

22. Ius T, Pauletto G, Isola M, Gregoraci G, Budai R, Lettieri $\mathrm{C}$, et al: Surgery for insular low-grade glioma: predictors of postoperative seizure outcome. J Neurosurg 120:12-23, 2014 
23. Kawaguchi T, Sonoda Y, Shibahara I, Saito R, Kanamori M, Kumabe T, et al: Impact of gross total resection in patients with WHO grade III glioma harboring the IDH 1/2 mutation without the 1p/19q co-deletion. J Neurooncol 129:505-514, 2016

24. Kim YH, Kim CY: Current surgical management of insular gliomas. Neurosurg Clin N Am 23:199-206, vii, 2012

25. Lang FF, Olansen NE, DeMonte F, Gokaslan ZL, Holland EC, Kalhorn C, et al: Surgical resection of intrinsic insular tumors: complication avoidance. J Neurosurg 95:638-650, 2001

26. Louis DN, Perry A, Reifenberger G, von Deimling A, Figarella-Branger D, Cavenee WK, et al: The 2016 World Health Organization Classification of Tumors of the Central Nervous System: a summary. Acta Neuropathol 131:803-820, 2016

27. Maffei A, Haley M, Fontanini A: Neural processing of gustatory information in insular circuits. Curr Opin Neurobiol 22:709-716, 2012

28. Mandonnet E, Martino J, Sarubbo S, Corrivetti F, Bouazza $S$, Bresson D, et al: Neuronavigated fiber dissection with pial preservation: laboratory model to simulate opercular approaches to insular tumors. World Neurosurg 98:239-242, 2017

29. Mehrkens JH, Kreth FW, Muacevic A, Ostertag CB: Long term course of WHO grade II astrocytomas of the insula of Reil after I-125 interstitial irradiation. J Neurol 251:14551464, 2004

30. Morshed RA, Young JS, Han SJ, Hervey-Jumper SL, Berger MS: Perioperative outcomes following reoperation for recurrent insular gliomas. J Neurosurg1-7, 2018

31. Moshel YA, Marcus JD, Parker EC, Kelly PJ: Resection of insular gliomas: the importance of lenticulostriate artery position. J Neurosurg 109:825-834, 2008

32. Neuloh G, Pechstein U, Schramm J: Motor tract monitoring during insular glioma surgery. J Neurosurg 106:582-592, 2007

33. Pallud J, Audureau E, Blonski M, Sanai N, Bauchet L, Fontaine D, et al: Epileptic seizures in diffuse low-grade gliomas in adults. Brain 137:449-462, 2014

34. Reil J: Die Sylvische Grube. Arch Physiol (Halle) 9:195_ 208, 1809

35. Ribas EC, Yagmurlu K, Wen HT, Rhoton AL Jr: Microsurgical anatomy of the inferior limiting insular sulcus and the temporal stem. J Neurosurg 122:1263-1273, 2015

36. Safaee MM, Englot DJ, Han SJ, Lawton MT, Berger MS: The transsylvian approach for resection of insular gliomas: technical nuances of splitting the Sylvian fissure. J Neurooncol 130:283-287, 2016

37. Sanai N, Mirzadeh Z, Berger MS: Functional outcome after language mapping for glioma resection. $\mathbf{N}$ Engl J Med 358:18-27, 2008

38. Sanai N, Polley MY, Berger MS: Insular glioma resection: assessment of patient morbidity, survival, and tumor progression. J Neurosurg 112:1-9, 2010

39. Simon M, Neuloh G, von Lehe M, Meyer B, Schramm J: Insular gliomas: the case for surgical management. J Neurosurg 110:685-695, 2009

40. Skrap M, Mondani M, Tomasino B, Weis L, Budai R, Pauletto $\mathrm{G}$, et al: Surgery of insular nonenhancing gliomas: volumetric analysis of tumoral resection, clinical outcome, and survival in a consecutive series of 66 cases. Neurosurgery 70:1081-1094, 2012

41. Smith JS, Chang EF, Lamborn KR, Chang SM, Prados MD, Cha S, et al: Role of extent of resection in the long-term outcome of low-grade hemispheric gliomas. J Clin Oncol 26:1338-1345, 2008
42. Southwell DG, Hervey-Jumper SL, Perry DW, Berger MS: Intraoperative mapping during repeat awake craniotomy reveals the functional plasticity of adult cortex. J Neurosurg 124:1460-1469, 2016

43. Śteňo A, Jezberová M, Hollý V, Timárová G, Šteňo J: Visualization of lenticulostriate arteries during insular low-grade glioma surgeries by navigated 3D ultrasound power Doppler: technical note. J Neurosurg 125:1016-1023, 2016

44. Tanriover N, Rhoton AL Jr, Kawashima M, Ulm AJ, Yasuda A: Microsurgical anatomy of the insula and the sylvian fissure. J Neurosurg 100:891-922, 2004

45. Türe U, Yaşargil MG, Al-Mefty O, Yaşargil DC: Arteries of the insula. J Neurosurg 92:676-687, 2000

46. Türe U, Yaşargil DC, Al-Mefty O, Yaşargil MG: Topographic anatomy of the insular region. J Neurosurg 90:720-733, 1999

47. Vanaclocha V, Sáiz-Sapena N, García-Casasola C: Surgical treatment of insular gliomas. Acta Neurochir (Wien) 139:1126-1135, 1997

48. Wang DD, Deng H, Hervey-Jumper SL, Molinaro AA, Chang EF, Berger MS: Seizure outcome after surgical resection of insular glioma. Neurosurgery 83:709-718, 2018

49. Wu A, Aldape K, Lang FF: High rate of deletion of chromosomes $1 p$ and $19 q$ in insular oligodendroglial tumors. J Neurooncol 99:57-64, 2010

50. Wu AS, Witgert ME, Lang FF, Xiao L, Bekele BN, Meyers CA, et al: Neurocognitive function before and after surgery for insular gliomas. J Neurosurg 115:1115-1125, 2011

51. Yaşargil MG, Reeves JD: Tumours of the limbic and paralimbic system. Acta Neurochir (Wien) 116:147-149, 1992

52. Yaşargil MG, von Ammon K, Cavazos E, Doczi T, Reeves JD, Roth P: Tumours of the limbic and paralimbic systems. Acta Neurochir (Wien) 118:40-52, 1992

53. Zentner J, Meyer B, Stangl A, Schramm J: Intrinsic tumors of the insula: a prospective surgical study of 30 patients. J Neurosurg 85:263-271, 1996

54. Zhuang DX, Wu JS, Yao CJ, Qiu TM, Lu JF, Zhu FP, et al: Intraoperative multi-information-guided resection of dominant-sided insular gliomas in a 3-T intraoperative magnetic resonance imaging integrated neurosurgical suite. World Neurosurg 89:84-92, 2016

\section{Disclosures}

The authors report no conflict of interest concerning the materials or methods used in this study or the findings specified in this paper.

\section{Author Contributions}

Conception and design: both authors. Acquisition of data: both authors. Analysis and interpretation of data: both authors. Drafting the article: both authors. Critically revising the article: both authors. Reviewed submitted version of manuscript: both authors. Approved the final version of the manuscript on behalf of both authors: Berger. Administrative/technical/material support: both authors. Study supervision: both authors.

\section{Correspondence}

Mitchel S. Berger: University of California, San Francisco, CA. mitchel.berger@ucsf.edu. 